\title{
Manipulação de Malhas 3D em uma aplicação de Realidade Aumentada utilizando o framework SXR
}

\author{
Adriano Gil, Afonso Costa, Juliana Figueira \\ SIDIA - Instituto de Ciência e Tecnologia \\ Manaus, Brasil \\ \{adriano.gil, afonso.costa, juliana.figueira\}@ sidia.com
}

\begin{abstract}
A Realidade Aumentada (RA) tem adquirido um apelo massivo à medida que novas frameworks vão surgindo possibilitando criar aplicações mais rapidamente. O SXR é um framework de código aberto da Samsung para desenvolver aplicações Android de realidade virtual e aumentada. Neste trabalho demonstramos a geração procedural de objetos em um mundo virtual a partir da manipulação de sua malha 3D.

Index Terms-augmented reality, 3D meshes, mobile
\end{abstract}

\section{INTRODUÇÃo}

O desenvolvimento de aplicativos para Realidade Aumentada (RA) tem se tornado bastante comum. Neste cenário, os desenvolvedores de aplicativos para a plataforma Android podem utilizar o framework SXR [1], o qual é simples, Open Source e construído especificamente para ser utilizado em dispositivos móveis. Logo, este tutorial visa demonstrar (através de exemplos práticos) a manipulação de malhas 3D em ambientes de RA utilizando o framework SXR. Nas próximas seções, são apresentados os conceitos básicos relacionados com: realidade aumentada, vértices, renderização, criação e manipulação de malhas 3D. Além disso, apresenta-se uma descrição de exemplo prático de manipulação de malhas $3 \mathrm{D}$ em ambiente RA.

\section{A. Realidade Aumentada}

Os conceitos de "realidade estendida" remetem a cenários futuristas da ficção: a plataforma de Minority Report, a sala de treinamento de X-man, até o monóculo Sayajin em Dragon Ball. Hoje, tanto a realidade virtual (VR) quanto a realidade aumentada são acessíveis até para dispositivos móveis. Celulares Samsung, por exemplo, podem fazer uso de um óculus adaptado para aplicações RV: o GearVR. Já aplicações RA não necessitamente necessariamente de nenhum equipamento especial.

Três critérios simples para uma aplicação ser considerada dentro da RA é que ela acrescente uma camada extra a realidade, seja interativa em tempo real e tridimensional de acordo com [2]. Esta camada pode remover, alterar ou acrescentar alguns elementos ao que é percebido sem o uso de dispositivos. Uma maneira simples de alcançar isso é utilizar a câmera de video de um smartphone para capturar o ambiente e ao apresentar esta captura na tela, acrescentar objetos virtuais interativos: personagens animados, caixas de texto, filtros de imagem, áudio espacializado, são exemplos do que pode ser explorado com RA.

\section{B. RA no $S X R$}

O SXR faz uso do ARCore [3] como biblioteca base no desenvolvimento de aplicativos em RA. Logo, as principais funcionalidades do ARCore (tais como: detecção de planos, posicionamento e tracking de objetos, luminosidade do ambiente) podem ser utilizadas pelo SXR.

\section{DemonstraçÃo DE UMA APLICAÇÃo SIMPLES EM RA}

A Figura 1 ilustra a detecção de planos (horizontal e vertical).

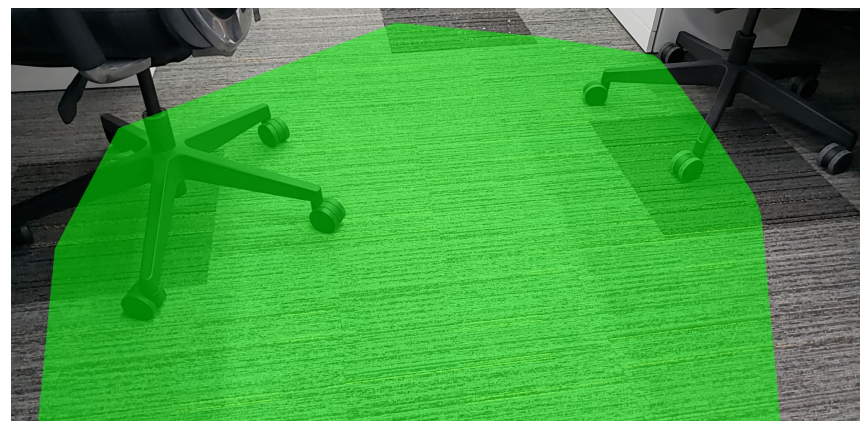

(a) Plano horizontal

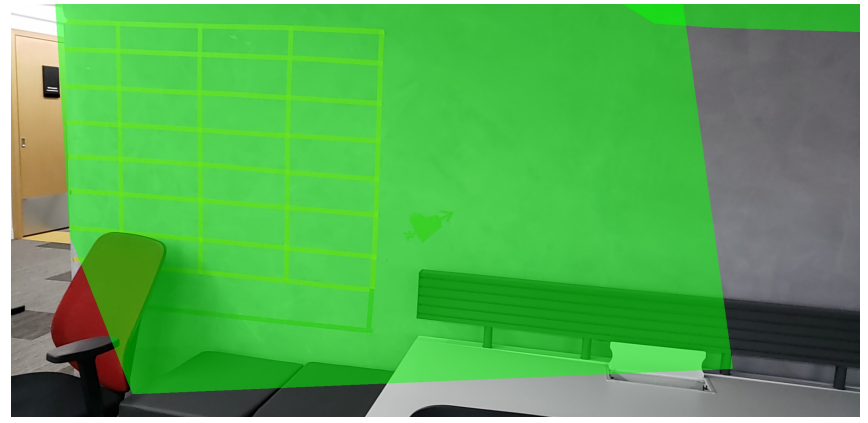

(b) Plano vertical

Fig. 1. Detecção de planos

Dependendo do tipo de aplicação que está sendo desenvolvida, a interação do usuário pode ser feita em plano horizontal e vertical. A Figura 2 mostra a interação do usuário com um plano horizontal, no qual um objeto 3D foi posicionado em um ponto do plano que o usuário escolheu.

A instanciação e renderização dos objetos 3D são feitas pela engine do SXR. Desta forma, torna-se possível utilizar animações dos objetos além de poder utilizar outras extensões 


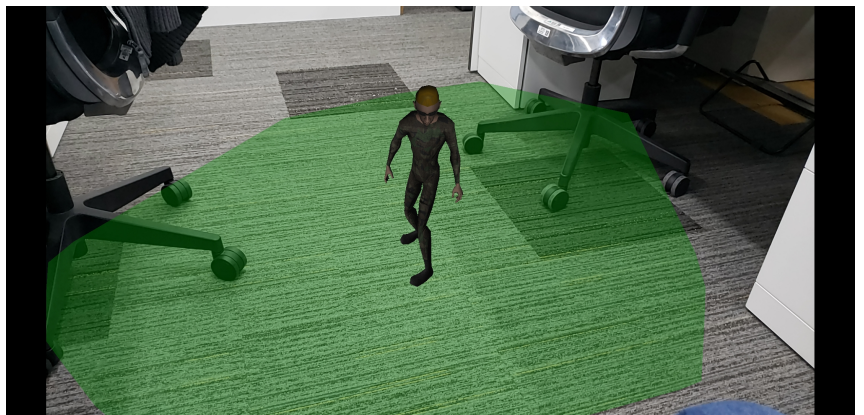

Fig. 2. Posicionamento de um objeto virtual (3D) em um plano detectado

do SXR, como por exemplo a extensão de física, na qual os objetos 3D podem ter características como massa, velocidade, atrito, etc.

\section{Malhas 3D}

O termo "Malhas 3D" é uma maneira de se referir a estruturas de dados para representação de malhas compostas por polígonos que compartilham vértices e arestas de modo a formar estruturas volumétricas, [4]. Para computação gráfica dessas malhas também é importante armazenar os dados de identificação dos vértices, faces, normais, grupos, texturas, coordenadas de textura e materiais do objeto.

\section{A. Conceitos básicos}

Para maior compreensão do desenvolvimento em ambientes de realidade estendida recomenda-se a revisão de álgebra e geometria para computação gráfica. Tópicos como operações com pontos, vetores e matrizes em diferentes sistemas de coordenadas: cartesiano, da mão direita e esquerda (z apontando para fora ou dentro), espacial (model space, world space, view space), quatérnios e diferentes representações de rotação (euler-angles, matrizes $3 \times 3$, eixo e ângulo, quatérnio, matriz $S Q T$ ). Por exemplo o capítulo 4 do livro 3D Math for Games [5].

\section{B. Geração procedural de objetos $3 D$}

Segundo [6], a geração procedural é uma área de inteligência artificial para jogos, onde artefatos de jogos são gerados utilizando algoritmos. Um exemplo clássico é o jogo .kkriger que com um executável de 64KB é capaz de gerar um gameplay completo de um jogo de tiro em primeira pessoa (first-person shooter - FPS), incluindo cenários 3D, armas, texturas, áudio, entre outros. Para criar objetos 3D proceduralmente é necessário a manipulação da malha 3D, alterando seus os vértices e triângulos que para compôr a forma desejada do objeto 3D.

\section{Introdução à Shaders}

Shaders são pequenos programas que rodam na placa gráfica (graphics processing unit - GPU) para processar uma grande carga de trabalho em paralelo e de maneira rápida. Esses programas podem ser incluídos em estágios específicos da pipeline gráfica dependendo do seu objetivo de forma a definir quais as cores de pixels que serão renderizadas na tela. A linguagem mais comum para shaders OpenGL é GLSL, propositalmente similar à linguagem $\mathrm{C}$. Os tipos mais comuns de programas são vertex (processamento de vértices) $\mathrm{e}$ fragment shader (após a rasterização processa uma cor e valor de profundidade), esses dois são o bastante para desenhar, manipular malhas e apresentar uma imagem de saída na pipeline do OpenGL [7].

\section{Manipulação de Malhas no SXR}

\section{A. Exemplos práticos}

Extrusão ou sweep translacional [8] é o processo de gerar uma superfície 3D a partir de uma plano e uma trajetória. Visualmente pode ser comparada ao alongamento de uma determinado plano em uma determinada direção. Esse processo pode ser implementado através da geração de novos vértices e triângulos à malha atual. Utilizando o framework SXR é possível desenvolver um algoritmo de geração procedural de uma malha 3D a partir da extrusão de uma superfície 3D visualizada em uma aplicação de realidade aumentada.

\section{Considerações Finais}

Neste tutorial foram apresentados os conceitos fundamentais necessários para a renderização de objetos 3D em realidade aumentada. A rasterização dos pixels da tela é possível através do uso de malhas 3D, uma estrutura de dados para descrever a geometria tridimensional. Então, shaders, código destinado para a GPU, são utilizados para configurar a visualização das formas geométricas. Utilizando esses conceitos é possível implementar uma aplicação de realidade aumentada para realizar extrusão de objetos $3 \mathrm{D}$ criados proceduralmente.

\section{REFERENCES}

[1] Samsung. (2018) Samsungxr. [Online]. Available: http://www.samsungxr.com/

[2] M. Billinghurst, A. Clark, G. Lee et al., "A survey of augmented reality," Foundations and Trends® in Human-Computer Interaction, vol. 8, no. 2-3, pp. 73-272, 2015.

[3] GoogleARCore. (2018) Arcore. [Online]. Available: https://developers.google.com/ar/develop/

[4] B. G. Baumgart, "A polyhedron representation for computer vision," in Proceedings of the May 19-22, 1975, national computer conference and exposition. ACM, 1975, pp. 589-596.

[5] J. Gregory, Game engine architecture. AK Peters/CRC Press, 2017.

[6] G. N. Yannakakis and J. Togelius, "A panorama of artificial and computational intelligence in games," IEEE Transactions on Computational Intelligence and AI in Games, vol. 7, no. 4, pp. 317-335, 2014.

[7] R. S. Wright Jr, N. Haemel, G. M. Sellers, and B. Lipchak, OpenGL SuperBible: comprehensive tutorial and reference. Pearson Education, 2010.

[8] E. José and D. S. dos Reis, "Implementação de um ambiente para modelagem de objetos 3d com uso de sweeping," Ph.D. dissertation, UNIVERSIDADE REGIONAL DE BLUMENAU, 2001. 